\title{
THE NAÏVE BAYES ALGORITHM FOR LEARNING DATA ANALYTICS
}

\author{
Tran Ngoc Viet \\ Faculty of Information Technology, Van Lang University \\ Ho Chi Minh City, Vietnam \\ viet.tn@vlu.edu.vn \\ Hoang Le Minh \\ Faculty of Information Technology, Van Lang University \\ Ho Chi Minh City, Vietnam \\ minh.hl@vlu.edu.vn \\ Le Cong Hieu \\ Faculty of Information Technology, Van Lang University \\ Ho Chi Minh City, Vietnam \\ hieu.lc@vlu.edu.vn \\ Tong Hung Anh \\ Faculty of Information Technology, Van Lang University \\ Ho Chi Minh City, Vietnam \\ anh.th@vlu.edu.vn
}

\begin{abstract}
The field of data science analysis in artificial intelligence is being deeply interested by many scientists around the world, many techniques are proposed to improve accuracy from Naive Bayes model by reducing the problem of interdependence between its attributes. The new research of this paper, which is presented step by step by the Naive Bayes (NB) method, is the method of applying NB with a new set of attributes. It is worthy of consideration that using learning data analytics method is receiving increased attention, because of the importance of learning data analytics, in order to provide predictive learning results of learners or offer better solutions to support schools to strengthen educational measures or have optimal plans to increase student retention rates studying at the school, as well as help students succeed in their studies. Data related to student management and training activities are collected from softwares, student affairs and learning management systems are operating in practice such as Edusoft.Net software, Moodle and Microsoft Teams, student attendance system by FaceID face recognition... Research, evaluate and select a number of new data technologies for the purpose of building student digital profile, including document storage functions, specifically intelligent functions such as creating, processing and storing documents.
\end{abstract}

Keywords: Machine learning; Naïve Bayes model; learning data analytics; algorithm; computational; complexity.

\section{Introduction}

In this article, we define learning data analytics by using the Naïve Bayes model (LDAB) with a new algorithm becomes a learning data analysis tool and lecturers can use the data in their courses to track and predict students with high performance. Finally, we discuss clarifying issues and concerns with the use of learning data analytics in higher education.

We are solving the problem of classifying data classes and creating datasets by automatic collection, preprocessing of data from Learning Management Systems such as Microsoft Teams, Moodle, E-learning in Van Lang University, Viet Nam for student's learning data analytics. We are working on a classification problem and have generated your set of hypothesis, created features and discussed the importance of variables. You have hundreds of thousands of data points and quite a few variables in your training data set. I would have used Naive Bayes model, which can be extremely fast relative to other classification algorithms. It works on Bayes theorem 
of probability to predict the class of unknown data sets. The basics of this algorithm, so that next time when you come across large data sets, you can bring this algorithm to action. Learning data analytics and dataset is defined as the measurement, collection, analysis, and reporting of data about learners and their contexts, for purposes of understanding and optimizing learning and the environments in which it occurs. Learning data analytics offers promise for predicting and improving student success and retention [13], [16] in part because it allows faculty, institutions, and students to make data-driven decisions about student success and retention.

The study covers how it detected the spam and non-spam messages using Naïve Bayes algorithm [3], [4]. This algorithm allows to handle large number of features. Naïve Bayes model support for handling large number words easily. The data set followed set of processes of machine learning in order to build the model. The data set acquired from the kaggle site and performed pre-processing using many methods including bag-of-words. Split the data into train and test model, it has built the model and evaluated. Hypothesize, create features and discuss the importance of variables from a set of big data. Big data such as hundreds of thousands of data points and quite a few variables in the set of training data. We use the Naive Bayes model which can process extremely fast compared to other categorical data analysis algorithms. Algorithm works based on Bayes theorem of probability to train data and predict classify of unknown set of data. The purpose of this paper is to provide a brief overview of learning data analytics and machine learning algorithms for learning data analytics. We will also explore its usage and applications, goals and examples.

\section{Naïve Bayes classifier}

Naive Bayes is a classification algorithm for multiclass classification problems. It is called Naive Bayes because the calculations of the probabilities for each class are simplified to make their calculations tractable.

Naive Bayes classifiers are built on Bayesian classification methods [4]. These rely on Bayes's theorem, equation describing the relationship of conditional probabilities of statistical quantities. In Bayesian classification, we're interested in finding the probability of a label given some observed features.

We can write as $P\left({ }^{E} / F\right)$, tells us how to express this in tern of quantities we can compute more directly

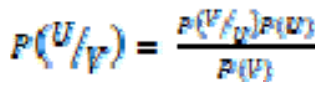

The Naïve Bayes classifier assigns to each instance the class value having the highest conditional probability.

Given a features vector $\mathbb{V}=\left(v_{\mathrm{L}}, v_{0}, \ldots, v_{\mathrm{n}}\right)$ and a class variable $U_{k}$, Bayes Theorem states that

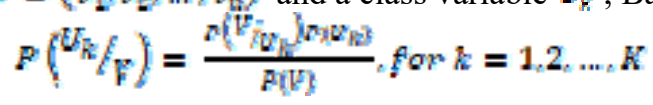

$P\left({ }^{U_{k}} / \mathrm{p}\right)$ the conditional probability that even $U_{k}$ occurs, given that $\nu$ has occurred. This is also known as the posterior probability.

$P\left(F_{U_{k}}\right)$ the conditional probability that even $V$ occurs, given that $U_{k}$ has occurre

$F\left(U_{k}\right)$ the prior probability of class

$P($ P) the prior probability of predictor

The likelihood $P\left(F^{F} / v_{k}\right)$ can be decomposed as

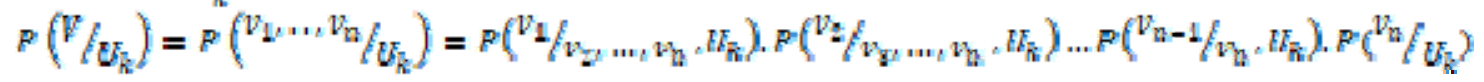

Multinomial Naive Bayes

$$
\begin{array}{r}
P\left(p \mid w_{1}, w_{n}\right) \propto P(v) P\left(w_{1}, w_{n} \mid v\right) \\
P\left(w_{k}, w_{n} \mid v\right)=\prod_{1} P(w, \mid v)
\end{array}
$$

Parameters: $P(w \mid v)$ for each document category $v$ and wordtype $w$

$P(v)$ prior distribution over document categories $v$

Learning: Estimate parameters as frequency ratios

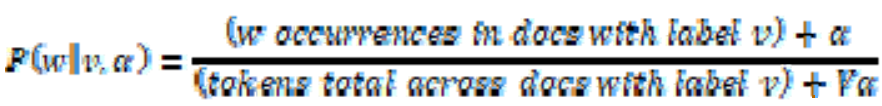

Predictions: Predict class

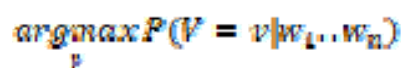




\section{Process Model}

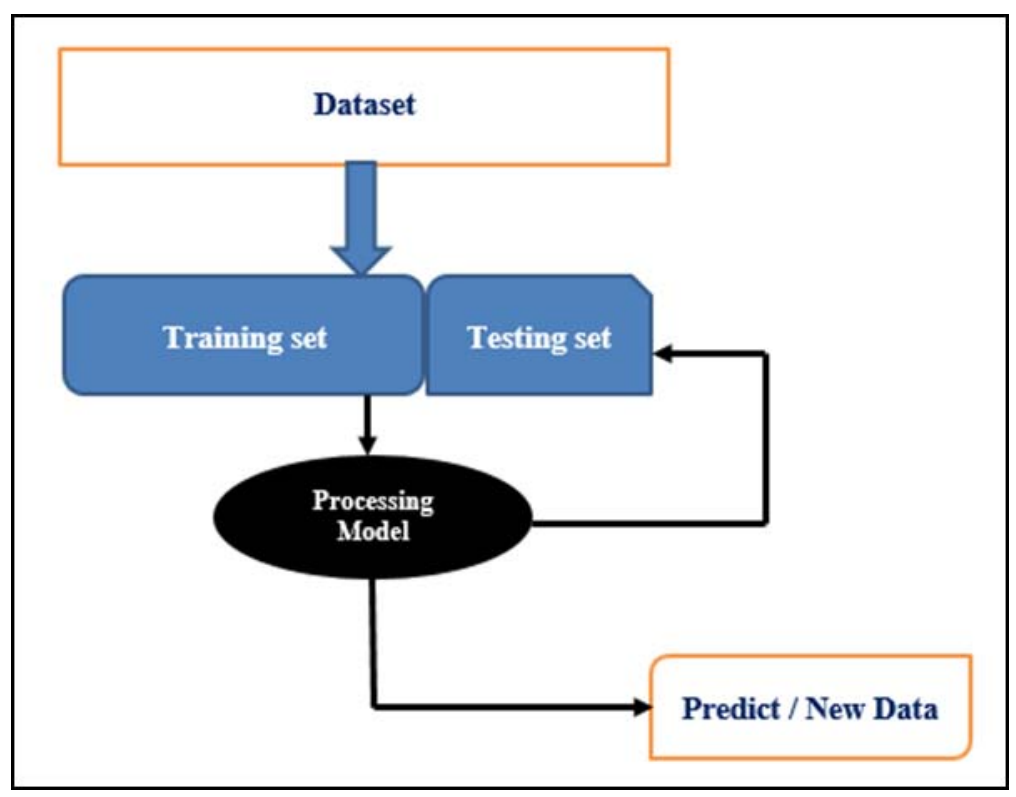

Fig. 1. A dataset is split into training and testing sets

\section{Steps LDAB Algorithm}

Data related to student management and training activities are collected from softwares, student affairs and learning management systems are operating in practice. New data technologies for the purpose of building student digital profile, including document storage functions, specifically intelligent functions such as creating, processing and storing documents.

Collected data has been processed, extracted according to the form.

Steps to build a basic Naive Bayes Model. Learning an LDAB is quite simple and mainly about esti-mating the parameters in the LDAB from the training data. The learning algorithm for LDAB is depicted as follows. Naive Bayes classifier in 4 easy steps, we will develop each piece of the algorithm in this section, then we will tie all of the elements together into a working implementation applied to a real dataset in the next section.

This Naive Bayes classifier is broken down into 4 parts

Input: A set Data of training examples

Output: An hidden Naive Bayes for Data, new set of data and evaluate the accuracy.

Student digital profile, probability results are calculated depending on the level of recommendation or warning to students about each student learning results.

Step 1: Gather the dataset/ Build a dataset frame.

Collect data by extracting from existing data storage such as MS Teams, FaceID or EduSoft.Net... Manage and organize data including deleting and removing unnecessary data.

Step 2: Create the model LDAB in Python (in this example LDAB).

Exploiting, processing data, presenting issues that need in-depth analysis and research.

Step 3: Predict using Test Dataset.

Analyze, train deeply data of the Naïve Bayes multi-class probability model and predict learning outcomes for the next learning period with accurate probability calculation;

Step 4: Prediction with a New Set of Dataset and evaluate the accuracy.

Report, transform and store the research results. 


\section{The Complexity of the Algorithm}

The independence assumption, Naive Bayes classifiers can quickly learn to use high dimensional features with limited training data compared to more sophisticated methods.

Let $n$ is number of training examples, $v^{*}$ is dimensionality of the features and $k$ is number of classes.

All it needs to do is computing the frequency of every feature value $v^{*}$ for each class, but space complexity of training is $\mathrm{O}\left(k \cdot v^{*} \cdot n\right)$ since you need to store the data which also takes time.

The computational complexity efficiency of Naive Bayes lies in the fact that the runtime complexity of Naive Bayes classifier is $\mathrm{O}\left(k \cdot v^{*} \cdot n\right)$.

\section{Applications of LDAB Algorithm}

\subsection{Computational}

$$
p\left(\mathrm{Y}^{\prime}\right)=\frac{3}{4^{\prime}} p(\mathrm{~N})=\frac{1}{4}
$$

\section{- TRAINING}

$\begin{array}{llllllll}\text { e1:x1 } & 2 & 1 & 2 & 2 & 2 & 2 & \\ \text { e2:x2 } & 2 & 2 & 2 & 1 & 2 & 2 & \\ \text { e3:x3 } & 2 & 2 & 2 & 2 & 2 & 2 & \mathrm{~d}=|\mathrm{V}|=6 \\ \text { Total } & 6 & 5 & 6 & 5 & 6 & 6 & =>\mathrm{N}_{\mathrm{Y}}=34 \\ \Rightarrow \lambda_{\mathrm{Y}} & 7 / 40 & 6 / 40 & 7 / 40 & 6 / 40 & 7 / 40 & 7 / 40 & 40=\mathrm{N}_{\mathrm{Y}}+|\mathrm{V}|\end{array}$

Class $\mathrm{Y}$

$\begin{array}{llllllll}\mathrm{e} 4: \mathrm{x} 4 & 0 & 0 & 1 & 0 & 2 & 1 & =>\mathrm{N}_{\mathrm{N}}=4 \\ \Rightarrow \lambda_{\mathrm{N}} & 1 / 10 & 1 / 10 & 2 / 10 & 1 / 10 & 3 / 10 & 2 / 10 & 10=\mathrm{N}_{\mathrm{N}}+|\mathrm{V}|\end{array}$

Class N

\section{- TEST}

$\mathrm{e} 5: \mathrm{x} 5=[1,1,2,1,2,1]$

$p(\mathrm{Y} \mid \mathrm{e} 5) \propto p(\mathrm{Y}) \cdot \prod_{i}^{d}=-2\left(x_{i} \mid \mathrm{Y}\right)=\frac{3}{4} \times \frac{7}{40} \times \frac{6}{40} \times\left(\frac{7}{40}\right)^{2} \times \frac{6}{40} \times\left(\frac{7}{40}\right)^{2} \times \frac{7}{40} \approx 4.8 \times 10^{-7}$

$p(\mathrm{~N} \mid \mathrm{e} 5) \propto p(\mathrm{~N}) . \Gamma_{i-1}^{d} p\left(x_{i} \mid \mathrm{N}\right)=\frac{1}{4} \times \frac{1}{10} \times \frac{1}{10} \times\left(\frac{2}{10}\right)^{2} \times \frac{1}{10} \times\left(\frac{3}{10}\right)^{2} \times \frac{2}{10} \approx 1.8 \times 10^{-7}$

$p(\mathrm{X} 5 \mid \mathrm{Y})>p(\mathrm{x} 5 \mid \mathrm{N}) \Rightarrow \mathrm{e} 5 \in \mathrm{Y}$

\section{Test data}

\subsection{Probability}

$$
\begin{aligned}
& p(\mathrm{Y} \mid \mathrm{e} 5)=\frac{4.8 \times 10^{-7}}{4.8 \times 10^{5}+1.8 \times 10} \approx 0.7273 \\
& \Rightarrow p(\mathrm{~N} \mid \mathrm{e} 5)=1-p(\mathrm{Y} \mid \mathrm{e} 5) \approx 0.2727 \text { and } e 5 \in Y .
\end{aligned}
$$

\subsection{Using the Naïve Bayes model in Python}

Supervised learning lets us make predictions based on the data that we see and thus apply generalisations

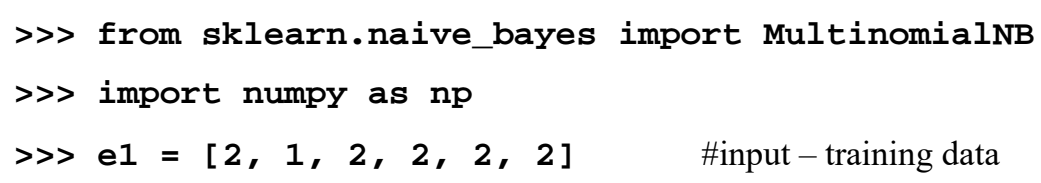




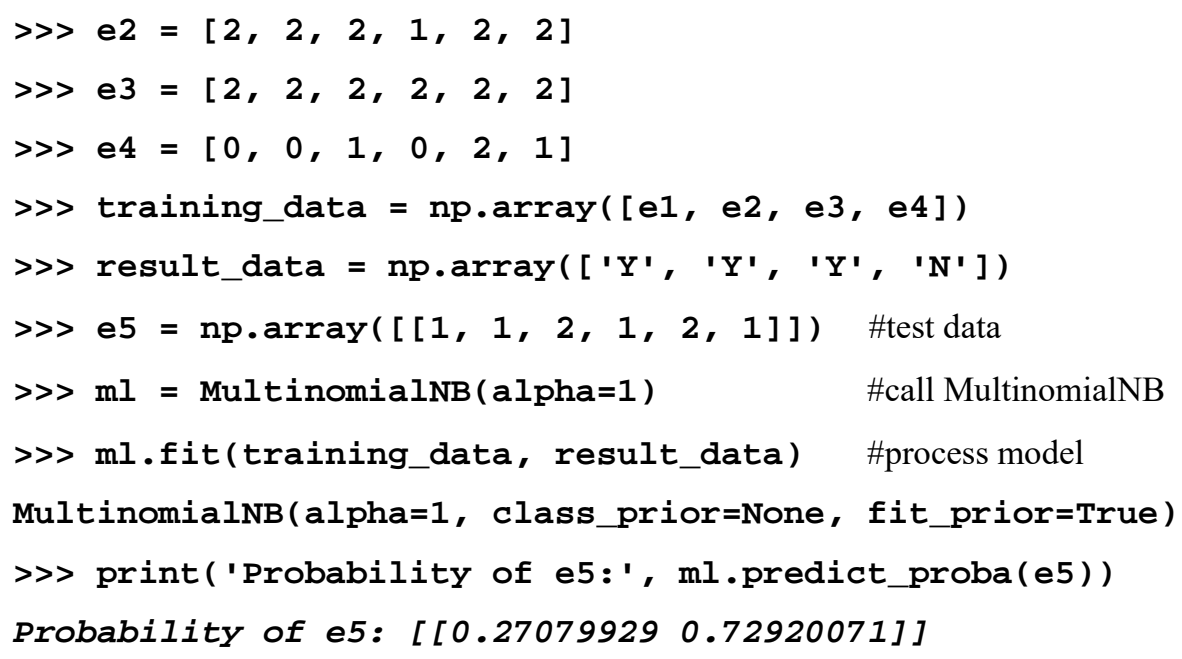

\#output - new set of data and evaluate the accuracy

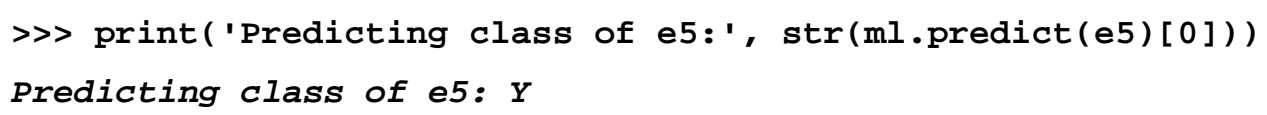

\section{Conclusion}

In this article, we build a model for learning data analytics from applying the Naïve Bayes probability formula with the purpose of modeling from real problems that can be applied accurately and effectively. Next, we propose to build an algorithm for learning data analytics and data needs to be tested correctly. Finally, a specific example is presented in detail to illustrate the LDAB algorithm as well as its complexity calculation.

\section{Acknowledgements}

The author would like to thank the Ministry of Technology - Higher Education and President, Van Lang University for financial support to this research.

\section{References}

[1] Webb, G.I., Boughton, J., Wang, Z (2005). Not so naive Bayes: Aggregating onedependence estimators. Machine Learning, 5-24.

[2] Witten, I.H., Frank, E (2000). Data mining: practical machine learning tools and techniques with Java implementations. San Francisco, CA: Morgan Kaufmann.

[3] A. S. Thanuja Nishadi (2019). Text Analysis: Naïve Bayes Algorithm using Python JupyterLab, International Journal of Scientific and Research Publications, Issue 11.

[4] Jake VanderPlas (2014). Frequentism and Bayesianism: A Python-driven Primer.

[5] Bhargava, K. Tara Phani (2018). Analysis and Design of Visualization of Educational Institution Database using Power BI Tool.

[6] Abdous, M., He, W., \& Yen, C (2012). Using data mining for predicting relationships between online question theme and final grade. Educational Technology \& Society, 15(3), 77-88.

[7] Brandon, K (2009). Investing in Education: The American Graduation Initiative. Office of SociaI Innovation and Civic Participation.

[8] Campbell, J. P., DeBlois, P. B., \& Oblinger (2007). Academic analytics: A new tool for a new era. EDUCAUSE Review, 42 (4), $40-57$.

[9] Dietz-Uhler, B., Hurn, J.E., \& Hurn (2012). Making use of data in an LMS to predict student performance: A learning analytics investigation. Unpublished manuscript.

[10] Dringus, L. P (2012). Learning analytics considered harmful. Journal of Asynchronous Learning Networks, 16(3), 87-100.

[11] Dyckhoff, A. L., Zielke, D., Bultmann, M., Chatti, M. A., \& Schroeder (2012). Design and implementation of a learning analytics toolkit for teachers. Educational Technology \& Society, 15(3), 58-76.

[12] Brain, D., Webb, G.I. (2002). The need for low bias algorithms in classification learning from large data sets. In: Proc. 16th European Conf. Principles of Data Mining and Knowledge Discovery (PKDD2002), Berlin:Springer-Verlag, 62-73.

[13] Dziuban, C., Moskal, P., Cavanaugh, T., \& Watts, A (2012). Analytics that inform the university: Using data you already have. Journal of Asynchronous Learning Networks, 16(3), 21-38.

[14] Greller, W. \& Drachslrer (2012). Translating learning into numbers: A generic framework for learning analytics.

[15] Ice, P., Diaz, S., Swan, K., Burgess, M., Sharkey, M., Sherrill, J., Huston, D., \& Okimoto, H (2012). The PAR framework proof of concept: Initial findings from a multi-institutional analysis of federated postsecondary data. Journal of Asynchronous Learning Networks, 16(3), 63-86.

[16] Long, P. \& Siemens, G (2011). Penetrating the fog: Analytics in Learning and Education.

[17] Webb, G.I. (2001). Candidate elimination criteria for lazy Bayesian rules. In: Proc. Fourteenth Australian Joint Conf. Artificial Intelligence. Volume 2256., Berlin:Springer, 545-556.

[18] Xie, Z., Hsu, W., Liu, Z., Lee, M.L.(2002). Snnb: A selective neighborhood based naive Bayes for lazy learning. In: Advances in Knowledge Discovery and Data Mining, Proc. Pacific-Asia Conference, Berlin:Springer, 104-114.

[19] Zhang, N. L (2004). Hierarchical latent class models for cluster analysis. Journal of Machine Learning Research 5:697-723. 
[20] Fayyad, U.M., Irani, K.B. (1993). Multi-interval discretization of continuous-valued attributes for classification learning. In: Proc. 13th Int. Joint Conf. Artificial Intelligence (IJCAI-93), Morgan Kaufmann, 1022-1029.

[21] Jones, S. J (2012). Technology review: The possibilities of learning analytics to improve learner centered decision making. The Community College Enterprise, Spring, 89-92.

[22] Zheng, Z., and Webb, G. I (2000). Lazy learning of bayesian rules. Journal of Machine Learning 41(1):53-84.

[23] Koller, D (2013). Probabilistic Graphical Models. Coursera Stanford - Lectures. Retrieved December 6, from https://class.coursera.org/pgm/lecture

[24] Koller, D., \& Friedman, N. (2009). Probabilistic graphical models: Principals and techniques MIT Press. ISBN 978-0262013192

[25] Koller, D., Friedman, N., Getoor, L., \& Taskar, B. (2007). Graphical models in a nutshell. In L. Getoor, \& B. Taskar (Eds.), An introduction to statistical relational learning () MIT Press.

[26] Friedman, N., Geiger, D., \& Goldszmidt, M. (1997). Bayesian network classifiers.

[27] Jurafsky, Dan and Manning, Christopher (2014). Natural Language Processing. Coursera Stanford - Lectures. Retrieved April 5, from https://class.coursera.org/nlp/lecture/28

[28] Naive Bayes for classifying Text (2014). Retrieved April 5 , from http://www.cs.nyu.edu/faculty/davise/ai/BayesText.html

[29] Chow, C.K. \& C.N. Liu (1968). Approximating discrete probability distributions with dependence trees. IEEE Trans. on Info. Theory, $14,462-467$.

\section{Authors Profile}
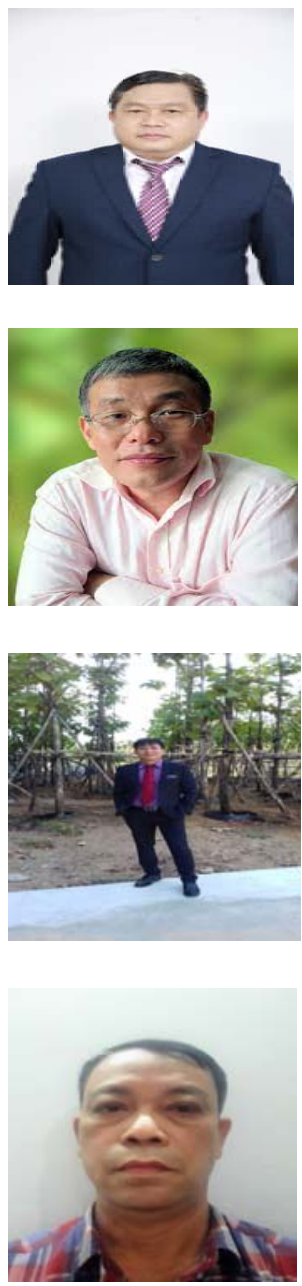

Dr. Tran Ngoc Viet. Born in 1973 in Thanh Khe District, Da Nang City, Vietnam. He graduated from Maths_IT faculty of Da Nang university of science. He got master of science at university of Danang and hold Ph.D Degree in 2017 at Danang university of technology. His main major: Applicable mathematics in transport, parallel and distributed process, data science, machine learning, graph theory and distributed programming.

Dr. Hoang Le Minh was born in 1957 in Ha Noi, Vietnam. He got Ph.D Degree in 1984 at Matxcova university of science. He is currently a lecturer at the Faculty of Information Technology at Van Lang University. His main major: Graph theory, data science, machine learning, big data analytics, data preprocessing and analysis, distributed programming.

Le Cong Hieu was born in 1979. He graduated from information technology faculty of Ho Chi Minh university of science. He has a master's degree in computer science, graduated in 2014 of Hue university. He is currently a lecturer at the Faculty of Information Technology at Van Lang University. His main major: discrete mathemetics, graph theory, machine learning and distributed programming.

Tong Hung Anh was born in 1964 in Hue, Vietnam. He has a master's degree in computer networking, graduated in 2011 university Paris VI. He is currently a lecturer at the Faculty of Information Technology at Van Lang university. His main major: Python for machine learning, distributed programming, mathemetics and statistics for data science, data pre-processing and analysis. 